\title{
Localization of collisionally inhomogeneous condensates in a bichromatic optical lattice
}

\author{
Yongshan Cheng ${ }^{1,2, *}$ and S. K. Adhikari ${ }^{1, \dagger}$ \\ ${ }^{1}$ Instituto de Física Teórica, UNESP - Universidade Estadual Paulista, 01.140-070 São Paulo, São Paulo, Brazil \\ ${ }^{2}$ Department of Physics, Hubei Normal University, Huangshi 435002, People's Republic of China
}

(Received 22 November 2010; published 28 February 2011)

\begin{abstract}
By direct numerical simulation and variational solution of the Gross-Pitaevskii equation, we studied the stationary and dynamic characteristics of a cigar-shaped, localized, collisionally inhomogeneous Bose-Einstein condensate trapped in a one-dimensional bichromatic quasiperiodic optical-lattice potential, as used in a recent experiment on the localization of a Bose-Einstein condensate [Roati et al., Nature (London) 453, 895 (2008)]. The effective potential characterizing the spatially modulated nonlinearity is obtained. It is found that the collisional inhomogeneity has influence not only on the central region but also on the tail of the Bose-Einstein condensate. The influence depends on the sign and value of the spatially modulated nonlinearity coefficient. We also demonstrate the stability of the stationary localized state by performing a standard linear stability analysis. Where possible, the numerical results are shown to be in good agreement with the variational results.
\end{abstract}

DOI: $10.1103 /$ PhysRevA.83.023620

PACS number(s): 03.75.Hh, 03.75.Lm, 67.85.Hj, 71.23.An

\section{INTRODUCTION}

Since Anderson predicted a localization of noninteracting electron wave in solids with a disorder potential 50 years ago [1], the Anderson localization has been observed and studied extensively in optics [2] and acoustics [3] and in Bose-Einstein condensates (BEC). In the study of Anderson localization in a BEC, disorder laser speckles [4] and quasiperiodic optical lattices (OL) [5] have been used. Random speckle potentials are produced when light is reflected by a rough surface or transmitted by a diffusive medium [6]. Billy et al. [4] observed the exponential tail of the spatial density distribution when a ${ }^{87} \mathrm{Rb}$ BEC was released into a one-dimensional (1D) waveguide in the presence of a controlled disorder created by a weak laser speckle. A bichromatic OL is realized by a primary lattice perturbed by a weak secondary lattice with incommensurate wavelength [7], and this system corresponds to an experimental realization of the Harper [8] or AubryAndré model [9]. Roati et al. [5] studied the localization of a noninteracting ${ }^{39} \mathrm{~K}$ BEC in a bichromatic OL. There have been many theoretical studies of localization using the numerical solution of the Gross-Pitaevskii (GP) equation [10] as well as using the Bose-Hubbard model [11] in addition to the experimental studies under different conditions on disorder [12]. There have been studies of localization in two and three dimensions [13] and of the destruction of localization with the increase of nonlinear repulsion $[14,15]$.

A Feshbach resonance (FR) driven by a magnetic [16] or optical [17] field allows one to vary the atomic interaction of a BEC in a controlled fashion [18], thus creating a noninteracting as well as a weakly interacting BEC for the study of Anderson localization. Fedichev et al. [19] predicted that the spatial variation of the laser field intensity by proper choice of the resonance detuning can lead to a spatial dependence of the atomic interaction, creating the so-called "collisionally inhomogeneous" BECs. The theoretical prediction has been demonstrated experimentally by Theis et al. with the ${ }^{87} \mathrm{Rb}$

\footnotetext{
*yong_shan@163.com

†adhikari@ift.unesp.br; URL [www.ift.unesp.br/users/adhikari]
}

BEC [17]. Sakaguchi and Malomed studied the formation of solitons in such BECs [20]. There have been studies of matterwave bright and dark solitons of the cubic-quintic nonlinear Schrödinger equation with time- and space-dependent nonlinearities [21] in a collisionally inhomogeneous environment, and of dynamical effects of a bright soliton BEC with local and smooth space variations of the two-body atomic scattering length [22]. There have been studies about how to introduce a space dependence in the nonlinear interaction of the BEC in a controlled way [23]. There have also been studies of soliton oscillations [24] and dynamical trapping and transport [25] in collisionally inhomogeneous BECs.

Here we combine the two interesting settings, namely, the bichromatic OL and a collisionally inhomogeneous BEC (with a spatially modulated nonlinearity), to study the statics and dynamics of a localized BEC in this setup. We assume that the spatial dependence of the nonlinearity, induced by the external magnetic field of the OL, has the same form as the bichromatic OL, i.e., the nonlinear coefficient in the GP equation is proportional to the OL intensity $[20,26]$. We study the effects of the spatially modulated nonlinearity on the shape of the density envelope, and the stability of the stationary localized states. The numerical results are shown to be in good agreement with the variational results, where applicable. On the other hand, the tail region of the stationary localized states is examined, where we find exponential decay in space indicating localization in a weak disorder potential. We study the location oscillation (oscillation of the center) and breathing oscillation of the localized states, induced by appropriate perturbation, employing numerical simulation of the GP equation.

In Sec. II we present a brief account of the 1D GP equation and the bichromatic OL potential used in our study, and a time-dependent variational analysis of the GP equation under appropriate conditions. We obtain a set of coupled evolution equations for the parameters of the localized state. The effective potential characterizing the spatially modulated nonlinearity is also described. In Sec. III we investigate the effects of the spatially modulated nonlinearity on the central and tail regions of a stationary localized BEC by a numerical solution of the GP equation using the split-step Fourier 
spectral method. For the central region, we demonstrate the stability of the localized states by performing a standard linear stability analysis. In Sec. IV the oscillation dynamics of a collisionally inhomogeneous BEC in a bichromatic OL is studied numerically. In Sec. V we present a brief discussion and concluding remarks.

\section{ANALYTICAL CONSIDERATION OF LOCALIZATION}

We consider a cigar-shaped quasi-1D BEC with inhomogeneous atomic interaction described by wave function $u(x, t)$ satisfying the following dimensionless $1 D$ GP equation $[27,28]$

$$
i \frac{\partial u}{\partial t}=-\frac{1}{2} \frac{\partial^{2} u}{\partial x^{2}}+g(x)|u|^{2} u+V(x) u,
$$

with normalization $\int_{-\infty}^{\infty}|u|^{2} d x=1$. The space variable $x$, time $t$, and energy are expressed in transverse harmonic oscillator units $a_{\perp}=\sqrt{\hbar /(m \omega)}, \omega^{-1}$ and $\hbar \omega$, where $m$ is the mass of an atom and $\omega$ is the angular frequency of the transverse trap. As in the experiment of Roati et al. [5], the potential $V(x)$ is taken to be a bichromatic OL of incommensurate wavelengths:

$$
V(x)=\sum_{l=1}^{2} A_{l} \sin ^{2}\left(k_{l} x\right),
$$

with $A_{l}=2 \pi^{2} s_{l} / \lambda_{l}^{2},(l=1,2)$, where $\lambda_{l}$ 's are the wavelengths of the OL potentials, $s_{l}$ 's are their intensities, and $k_{l}=2 \pi / \lambda_{l}$ the corresponding wave numbers. In this investigation, we take the incommensurate ratio of the two components [29] $k_{2} / k_{1}=(\sqrt{5}-1) / 2 \approx 0.618033989 \ldots$ In the actual experiment of Roati et al. [5], however, the parameter is $k_{2} / k_{1}=1.1972 \ldots$. Without losing generality, we further take $\lambda_{1}=10$, and $s_{1}=10, s_{2}=0.3 s_{1}$ which is roughly the same ratio $s_{2} / s_{1}$ as in the experiment of Roati et al. [5].

By means of the FR technique controlled by properly designed configurations of external optical fields [20,26], the spatial variation of laser-field intensity $I(x)$ produces the spatial variation of the atomic scattering length. As the potential $V(x)$ is also proportional to laser-field intensity, it is reasonable to assume the spatial dependence of the atomic scattering length is similar to $V(x)$. Then, the atomic scattering length can be given as $a_{s}=a_{s 0}+c V(x)$; here $V(x)$ is the same as Eq. (2), $a_{s 0}$ is scattering length of the corresponding collisionally homogeneous system, and $c$ is a constant coefficient related to the optical intensity and may be either positive or negative. Thus, the nonlinear coefficient $g(x)$ in Eq. (1) has a spatial dependence of the form

$$
g(x)=\varepsilon_{0}+\varepsilon \sum_{l=1}^{2} A_{l} \sin ^{2}\left(k_{l} x\right) .
$$

The nonlinearity $\varepsilon_{0}$ is given by [27] $\varepsilon_{0}=2 a_{s 0} N / a_{\perp}$ with $N$ the number of atoms, and $\varepsilon=2 c N / a_{\perp}$ is the spatially modulated nonlinearity coefficient. Because the intensity-independent nonlinear coefficient $\varepsilon_{0}$ may be altered independently of the other parameters [18], in order to focus our attention on the effect of the spatially modulated nonlinearity on the localization of the BEC, we let $\varepsilon_{0}=0$ in the following.

Usually, the BEC localized states formed on a bichromatic lattice may occupy many sites of the OL potential $[5,30]$. For certain values of the parameters, however, potential (2) leads to localized states confined practically to a single site of the OL potential. When this happens, a variational approach for solving the GP equation is useful. To apply the variational approach to the localized BEC, we adopt the following variational ansatz:

$$
\begin{aligned}
u(x, t)= & \frac{1}{\pi^{1 / 4}} \sqrt{\frac{N}{w}} \exp \left[-\frac{\left(x-x_{0}\right)^{2}}{2 w^{2}}\right] \\
& \times \exp \left\{i\left[\gamma\left(x-x_{0}\right)+\beta\left(x-x_{0}\right)^{2}+\phi\right]\right\},
\end{aligned}
$$

with $w$ as the spatial width of the localized state centered at $x_{0}, \gamma$ is the linear phase coefficient, $\beta$ is the chirp,and $\phi$ is the phase. These are time-dependent parameters [30]. The Lagrangian of the system is [31,32]

$$
\begin{aligned}
L(t)= & \int_{-\infty}^{\infty}\left[\frac{i}{2}\left(u^{*} \frac{\partial u}{\partial t}-u \frac{\partial u^{*}}{\partial t}\right)-\frac{1}{2}\left|\frac{\partial u}{\partial x}\right|^{2}\right. \\
& \left.-\frac{1}{2} g(x)|u|^{4}-V(x)|u|^{2}\right] d x \\
= & N\left(\gamma \dot{x}_{0}-\frac{1}{2} \dot{\beta} w^{2}-\dot{\phi}\right)-\frac{N}{2}\left(\frac{1}{2 w^{2}}+\gamma^{2}+2 \beta^{2} w^{2}\right) \\
& +N^{2} L_{M}+N L_{V},
\end{aligned}
$$

where the overhead dot denotes time derivative, the star denotes complex conjugation, and

$$
\begin{array}{r}
L_{M}=\frac{\varepsilon}{4 \sqrt{2 \pi} w} \sum_{l=1}^{2} A_{l}\left[\cos \left(2 k_{l} x_{0}\right) \exp \left(-\frac{k_{l}^{2} w^{2}}{2}\right)-1\right], \\
L_{V}=-\frac{1}{2} \sum_{l=1}^{2} A_{l}\left[1-\cos \left(2 k_{l} x_{0}\right) \exp \left(-k_{l}^{2} w^{2}\right)\right] .
\end{array}
$$

We use the variational Euler-Lagrange equation

$$
\frac{\partial L}{\partial \sigma}-\frac{d}{d t} \frac{\partial L}{\partial \dot{\sigma}}=0
$$

where the variational parameters are $\sigma=\phi, x_{0}, \gamma, \beta$, and $w$. The first variational equation using $\sigma=\phi$ yields $N=$ constant. We take this constant to be unity and use it in the subsequent equations. The other choices of $\sigma$ respectively lead to the following equations:

$$
\begin{gathered}
\dot{\gamma}=-\frac{\varepsilon}{2 \sqrt{2 \pi} w} \sum_{l=1}^{2} A_{l} k_{l} \sin \left(2 k_{l} x_{0}\right) \exp \left(-\frac{1}{2} k_{l}^{2} w^{2}\right) \\
-\sum_{l=1}^{2} A_{l} k_{l} \sin \left(2 k_{l} x_{0}\right) \exp \left(-k_{l}^{2} w^{2}\right), \\
\dot{x}_{0}=\gamma, \\
\dot{w}=2 \beta w \equiv F(w, \beta), \\
\dot{\beta}=\frac{1}{2 w^{4}}-2 \beta^{2}+\frac{1}{w} \frac{\partial L_{M}}{\partial w}+\frac{1}{w} \frac{\partial L_{V}}{\partial w} \equiv G(w, \beta) .
\end{gathered}
$$

The Hamiltonian of the BEC is

$$
\begin{aligned}
H & =\dot{x}_{0} \frac{\partial L}{\partial \dot{x}_{0}}+\dot{\phi} \frac{\partial L}{\partial \dot{\phi}}+\dot{\beta} \frac{\partial L}{\partial \dot{\beta}}-L \\
& =\frac{1}{2}\left(\frac{1}{2 w^{2}}+\gamma^{2}+2 \beta^{2} w^{2}\right)-L_{M}-L_{V} .
\end{aligned}
$$


Equations (11) and (12) determine the evolution of the width $w$ once $x_{0}$ is known. Equations (9) and (10) determine the evolution of center $x_{0}$ once the width $w$ is known. To study the dynamics of the localized state, we insert Eq. (9) into Eq. (10) to get an anharmonic effective potential $V_{\text {eff }}$ :

$$
\begin{gathered}
\frac{d^{2} x_{0}}{d t^{2}}=-\frac{\partial V_{\mathrm{eff}}}{\partial x_{0}} \equiv-\frac{\partial}{\partial x_{0}}\left(V_{\mathrm{effM}}+V_{\mathrm{effV}}\right), \\
V_{\mathrm{effM}}=\frac{-\varepsilon}{4 \sqrt{2 \pi} w} \sum_{l=1}^{2} A_{l} \cos \left(2 k_{l} x_{0}\right) \exp \left(-\frac{k_{l}^{2} w^{2}}{2}\right), \\
V_{\mathrm{effV}}=-\frac{1}{2} \sum_{l=1}^{2} A_{l} \cos \left(2 k_{l} x_{0}\right) \exp \left(-k_{l}^{2} w^{2}\right) .
\end{gathered}
$$

The effective potential has two terms. The second term on the right-hand side of Eq. (14), $V_{\text {effv }}$, arises from the bichromatic OL and contributes to an attractive well, if $\left|x_{0}\right|$ is small enough. The first term, $V_{\text {effM }}$, is induced by the spatial modulation of the nonlinearity and is actually a pseudopotential [33]. The pseudopotential is quasiperiodic and is a potential barrier or well depending on the sign and value of the coefficient $\varepsilon$.

\section{STATIONARY LOCALIZED STATE}

The stationary states are obtained by setting the time derivative in Eqs. (9)-(12) to zero. Then the simplest solution of Eqs. (9) and (10) is $x_{0}=0$ and we consider below an immobile localized state at origin $\left(x_{0}=0\right)$ with $\gamma=0$. Equations (11) and (12) determine $w$ and can be written as

$$
\begin{gathered}
\beta_{0}=0 \\
1+\frac{\varepsilon w_{0}}{2 \sqrt{2 \pi}} \sum_{l=1}^{2} A_{l}\left[1-\left(1+k_{l}^{2} w_{0}^{2}\right) \exp \left(-\frac{1}{2} k_{l}^{2} w_{0}^{2}\right)\right] \\
-2 w_{0}^{4} \sum_{l=1}^{2} A_{l} k_{l}^{2} \exp \left(-k_{l}^{2} w_{0}^{2}\right)=0
\end{gathered}
$$

The effective potential felt by a stationary localized state at $x=x_{0}=0$ is obtained from Eqs. (14)-(16) and is plotted in Fig. 1 where the width $w$ is obtained by solving numerically Eq. (18). With the increase of $\varepsilon$, the strength of both disorder and the quasiperiodic effective potential increases.

To understand the effects of the coefficient $\varepsilon$, we obtain the stationary localized states by solving Eq. (1) numerically with real-time split-step Fourier spectral method with a space step 0.04 and time step 0.0001 . We checked the accuracy of the results by varying the space and time steps and the total number of space and time steps. To compare with numerics, the variational width is obtained by solving Eq. (18).

Typical numerical and variational results for the density, width, and Hamiltonian of the localized state at $x=0$ are exhibited in Fig. 2 for $-1 \leqslant \varepsilon \leqslant 1$. The variational Hamiltonian is obtained from Eq. (13) and the numerical Hamiltonian is obtained from $H=\int_{-\infty}^{\infty}\left[\left|u^{\prime}\right|^{2} / 2+g(x)|u|^{4} / 2+V(x)|u|^{2}\right] d x$. Figures 2(a) and 2(b) exhibit the density of the stationary localized states corresponding to $\varepsilon=-1$ and 1 , respectively. Figures 2(c) and 2(d) exhibit the variation of $w$ and $H$

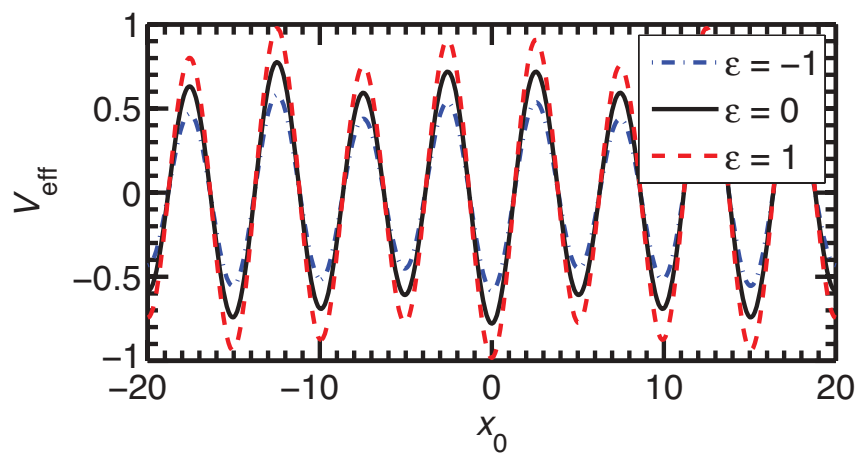

FIG. 1. (Color online) The dimensionless effective potentials $V_{\text {eff }}$ "felt" by an immobile localized state for different $\varepsilon$ from Eqs. (15) and (16) where the width $w$ is calculated from Eq. (18).

with $\varepsilon$. The numerical width $w$ in Fig. $2(\mathrm{c})$ is $\sqrt{2}$ times the root-mean-square (rms) size of the BEC. Figure 2 indicates that the numerical results are in good agreement with the variational results. Figure 2(c) shows that the width decreases and Fig. 2(d) shows that the Hamiltonian increases with the change of $\varepsilon$ from negative to positive. The dependence of the variational width on $\varepsilon$ can be qualitatively understood as follows. The height of the central well of the effective potential increases with increasing $\varepsilon$ as shown in Fig. 1. Hence the central part of the localized state with a Gaussian shape becomes narrow with the increase of $\varepsilon$, as can be seen from Figs. 2(a) and 2(b). The variational Gaussian ansatz only represents this central part and hence the variational width decreases with increasing $\varepsilon$. However, the numerical width (rms size) shown in Fig. 2(c) receives nontrivial contributions from both the central Gaussian part and the extended exponential tail of the wave function [viz. Fig. 4(a)], making it difficult to predict even qualitatively the variation of the numerical width with $\varepsilon$. It is interesting to compare these results with those of a collisionally homogeneous condensate where a constant
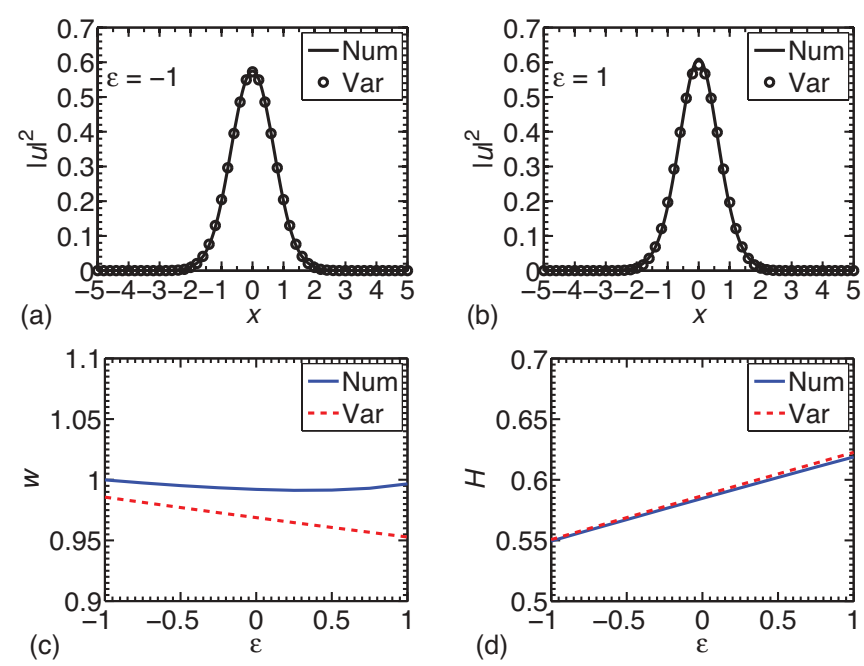

FIG. 2. (Color online) Numerical (lines) and variational (chain of symbols) densities $|u|^{2}$ of the localized BEC versus $x$ for (a) $\varepsilon=-1$, (b) $\varepsilon=1$. (c) Numerical (solid lines) and variational (dashed lines) dimensionless widths $w$ versus $\varepsilon$ and (d) Hamiltonian versus $\varepsilon$. (All quantities are dimensionless.) 
negative (attractive) nonlinearity leads to a reduction of the width and a constant positive (repulsive) nonlinearity increases the width of the localized state [15]. The numerical width is larger than the variational width in Fig. 2(c) due to the long exponential tail of the numerically obtained BEC (viz., Fig. 4). Also, the difference between the variational and numerical widths increases for larger values of $\varepsilon$, because for larger $\varepsilon$ the exponential tail is more pronounced resulting in a larger width (viz., Fig. 4).

It is important to investigate the stability of the stationary state governed by Eqs. (17) and (18) against perturbation by a standard linear stability analysis. Introducing small fluctuations around the stationary solution $\left(w_{0}, \beta_{0}\right), w^{\prime}(t)=w(t)-$ $w_{0}, \beta^{\prime}(t)=\beta(t)-\beta_{0}$, and linearizing Eqs. (11) and (12) indicated by them, a set of two linear equations are obtained:

$$
\begin{gathered}
\frac{d w^{\prime}(t)}{d t}=F_{w}\left(w_{0}, \beta_{0}\right) w^{\prime}(t)+F_{\beta}\left(w_{0}, \beta_{0}\right) \beta^{\prime}(t), \\
\frac{d \beta^{\prime}(t)}{d t}=G_{w}\left(w_{0}, \beta_{0}\right) w^{\prime}(t)+G_{\beta}\left(w_{0}, \beta_{0}\right) \beta^{\prime}(t),
\end{gathered}
$$

where the subscripts $w$ and $\beta$ denote a derivative with respect to the respective variable. Assuming the solution of $w^{\prime}(t)$ and $\beta^{\prime}(t)$ in exponential form, $\sim \exp (\mathcal{E} t)$, the eigenvalue $\mathcal{E}$ is

$$
\begin{aligned}
2 \mathcal{E}= & F_{w}\left(w_{0}, \beta_{0}\right)+G_{\beta}\left(w_{0}, \beta_{0}\right) \\
& \pm\left\{\left[F_{w}\left(w_{0}, \beta_{0}\right)+G_{\beta}\left(w_{0}, \beta_{0}\right)\right]^{2}\right. \\
& \left.+4 F_{\beta}\left(w_{0}, \beta_{0}\right) G_{w}\left(w_{0}, \beta_{0}\right)\right\}^{1 / 2} .
\end{aligned}
$$

From Eqs. (11) and (12), we find $F_{w}\left(w_{0}, \beta_{0}\right)=0, G_{\beta}\left(w_{0}\right.$, $\left.\beta_{0}\right)=0$, and

$$
\begin{gathered}
F_{\beta}\left(w_{0}, \beta_{0}\right)=2 w_{0} \\
G_{w}\left(w_{0}, \beta_{0}\right)=-\frac{2}{w_{0}^{5}}+2 w_{0} \sum_{l=1}^{2} A_{l} k_{l}^{4} \exp \left(-k_{l}^{2} w_{0}^{2}\right) \\
+\frac{\varepsilon}{4 \sqrt{2 \pi} w_{0}^{4}} \sum_{l=1}^{2} A_{l}\left[-3+\left(3+2 k_{l}^{2} w_{0}^{2}+k_{l}^{4} w_{0}^{4}\right)\right. \\
\left.\times \exp \left(-\frac{1}{2} k_{l}^{2} w_{0}^{2}\right)\right]
\end{gathered}
$$

which leads to the eigenvalues

$$
\mathcal{E}= \pm\left[F_{\beta}\left(w_{0}, \beta_{0}\right) G_{w}\left(w_{0}, \beta_{0}\right)\right]^{1 / 2} .
$$

To investigate the stability, Eq. (18) is first solved to get $w_{0}$ as a function of $\varepsilon$. This result is then inserted in Eqs. (22) and (23) to get $F_{\beta}\left(w_{0}, \beta_{0}\right)$ and $G_{w}\left(w_{0}, \beta_{0}\right)$. The graphical representation of the two functions is shown in Figs. 3(a) and $3(\mathrm{~b})$. In the case of a small coefficient $\varepsilon$, we can find that $G_{w}\left(w_{0}, \beta_{0}\right)<0$ and $F_{\beta}\left(w_{0}, \beta_{0}\right)=2 w_{0}>0$. Thus, both the eigenvalues from Eq. (24) must be imaginary, and the localized state from Eqs. (17) and (18) is stable against small perturbation.

Anderson localization in a weakly disordered potential is characterized by a long exponential tail of the localized state. For collisionally homogeneous condensates, the experimental [5] and theoretical [15] investigations have demonstrated that the localized BECs have an exponential tail for weakly

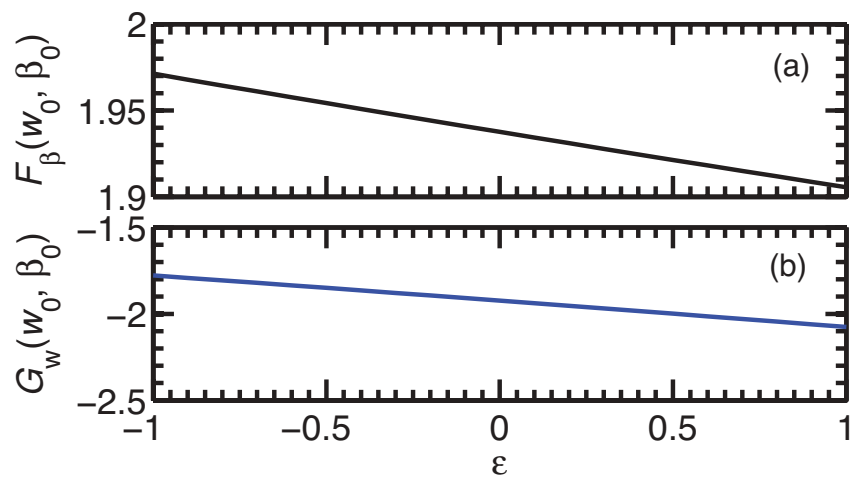

FIG. 3. (Color online) (a) The dimensionless functions $F_{\beta}\left(w_{0}, \beta_{0}\right)$ and (b) $G_{w}\left(w_{0}, \beta_{0}\right)$ versus $\varepsilon$. It shows that the signs of the two functions are opposite for $-1 \leqslant \varepsilon \leqslant 1$.

interacting or noninteracting BEC in a quasiperiodic OL. In order to observe the effect of spatially modulated nonlinearity on the tail region, we plot in Fig. 4(a) the density distribution $|u|^{2}$ of the stationary BEC on log scale. As we see, the long exponential tail extends from $x \approx \pm 2$ to $x \approx \pm 20$, whereas the central part of density for $|x|<2$ represents a Gaussian distribution. By an exponential fitting of the exponential function $\sim \exp (-|x| / L)$ to the tails of density distribution, the localization length $L$ vs $\varepsilon$ is illustrated in Fig. 4(b) which shows that $L$ increases nonlinearly with $\varepsilon$. An increase in $\varepsilon$ represents a decrease in disorder thus resulting in larger values of localization length.

\section{DYNAMICS OF LOCALIZED STATE}

To get further insight into the effects of the spatially modulated nonlinearity $\varepsilon$ on the localized states, we now study some dynamics of the localized state. First, we study numerically the oscillation of the localized states in an external potential. According to Eqs. (14)-(16), the motion of the localized BEC can be approximately regarded as that of a particle inside an effective potential $V_{\text {eff }}$. Because of the exponential tail and elasticity of the localized state, although the variational results may not be good for the dynamical evolution, they can provide a qualitative physical understanding of the dynamics using the effective potential. To study the motion of the localized state, first we create a stationary localized BEC with spatially modulated nonlinearity in the bichromatic OL. Successively,
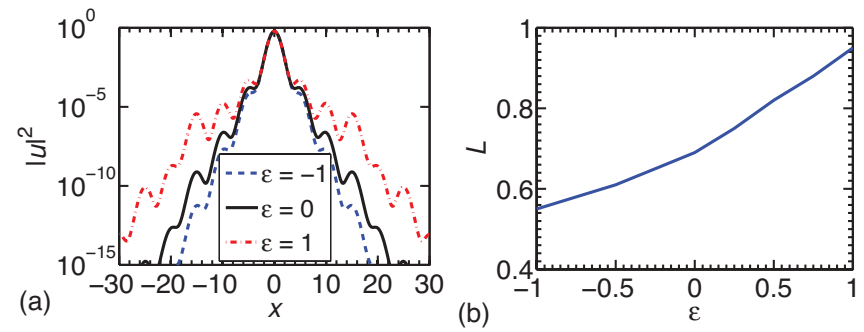

FIG. 4. (Color online) (a) The numerically obtained dimensionless density $|u|^{2}$ vs dimensionless $x$ for different $\varepsilon$. (b) The dimensionless localization lengths $L$ vs $\varepsilon$. The localization length $L$ is obtained by exponential fitting to the tails of density distribution with $\sim \exp (-|x| / L)$. 


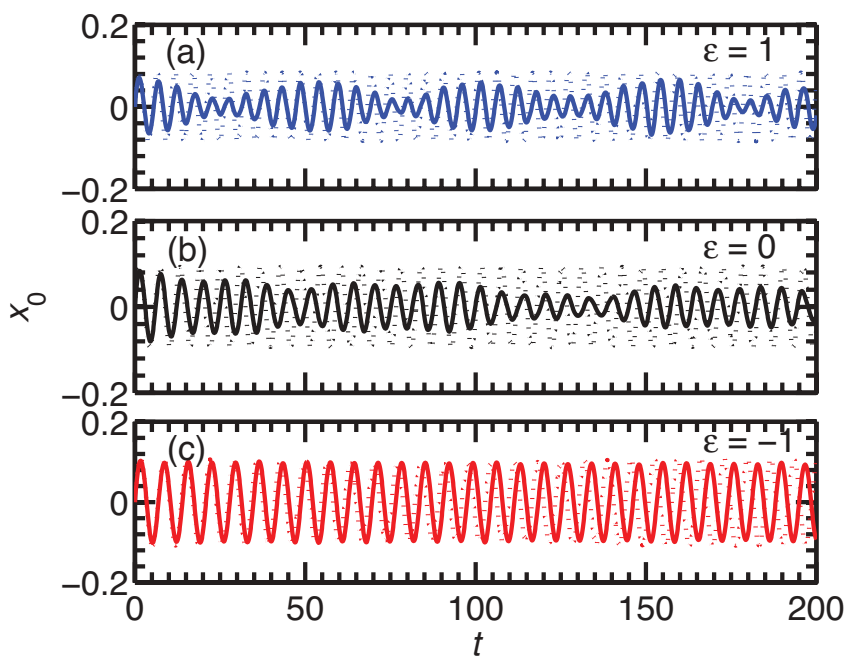

FIG. 5. (Color online) The center of the localized state $x_{0}$ vs time $t$ (both dimensionless) during the location oscillation initiated by suddenly introducing an initial momentum $p_{0}=0.1$ by the transformation $u \rightarrow u_{0} \exp \left(i p_{0} x\right)$ for $\varepsilon=$ (a) 1 , (b) 0 , and (c) -1 : numerical result (full line) and variational result (dotted line).

at $t=0$, we suddenly introduce an initial momentum $p_{0}=0.1$ by $u(x) \rightarrow u_{0}(x) \exp \left(i p_{0} x\right)$, where $u_{0}$ is the wave function of the stationary localized BEC. From the experimental point of view, the initial momentum can be given by suddenly moving the OL [34]. It is found that after the perturbation, the density envelope suffers an abrupt change but remains localized. Actually, the localized BEC is an elastic object and not a rigid one. Hence, both the center and the density distribution of the localized state perform oscillations.

The evolution of the center $x_{0}$ of the localized BEC as obtained from numerical simulation (full line) is shown in Fig. 5 where $x_{0}$ is obtained by an instant Gaussian-function fitting to the central region of the density distribution. The variational results are also shown in this figure. The top, middle, and bottom panels correspond to $\varepsilon=1,0$, and -1 , respectively. We find that, in general, the oscillation of the localized state could be quasiperiodic after an initial damping, which can be explained on the basis of energy conservation. Because of the deformation of the density envelope, a part of the initial kinetic energy is converted into elastic energy of deformation and this leads to the damped oscillation. This deformation will be larger for large $\varepsilon(=1)$, when the localized state is loosely bound with large exponential tail [viz. Fig. 4(a)]. Consequently, an oscillation of the center $x_{0}$ with rapidly varying amplitude is found denoting easy periodic transfer of energy between location oscillation and deformation. For small $\varepsilon(=-1)$, the localized state is more compact and tightly bound, so that it can be treated like a rigid object. The exchange of energy is less probable in that case, and a periodic oscillation of the center $x_{0}$ with constant amplitude is found. Eventually, the energy of deformation is liberated leading to an increase in the amplitude of oscillation. During the subsequent oscillation cycle, the conversion between the kinetic energy and elastic strain energy causes the quasiperiodical movement of the localized state. In the variational formulation the exchange of kinetic energy to the energy of deformation is not allowed, and the resultant oscillation is of a fixed amplitude without damping. Nevertheless, the numerical frequency of location oscillation is in agreement with the variational frequency within an estimated error of about $2.5 \%$. As pointed out in Sec. III, a positive $\varepsilon$ leads to a tighter trapping and vice versa. Then, with the same initial velocity, the tighter trapping causes the localized state to oscillate with larger frequency and smaller amplitude, and a weaker trapping leads to a smaller frequency and larger amplitude of oscillation as indicated in the numerical results of Fig. 5. The variational frequency follows the same trend as $\varepsilon$ is changed from positive values to negative values.

Next we consider a breathing oscillation of the localized BEC, started by suddenly changing the strength of the secondary lattice $s_{2}$ from 3 to 3.5 at $t=0$. We investigate how the breathing oscillation of the localized BEC is changed by the spatially modulated nonlinearity. Then, the nonlinearity in Eq. (1), $g(x)$, also changes with the new OL potential. We present numerical results in Figs. 6(a), 6(b), and 6(c) for the time evolution of the width $w$ of the localized state for $\varepsilon=1,0$, and -1 , respectively. The variational equations (11) and (12) were solved to obtain the oscillation of the central part, and this result is shown in Fig. 6(d) for $\varepsilon=0$. (The Gaussian variational ansatz without any exponential tail only carries information about the central part.) There are two regions of the localized state which oscillate with two distinct frequencies: (i) the central region with Gaussian distribution and (ii) the outer tail with exponential distribution. The net result is the harmonic oscillation of the width with a small frequency and large amplitude due to the oscillation of the exponential tail, which is modulated by rapid oscillation coming from the central

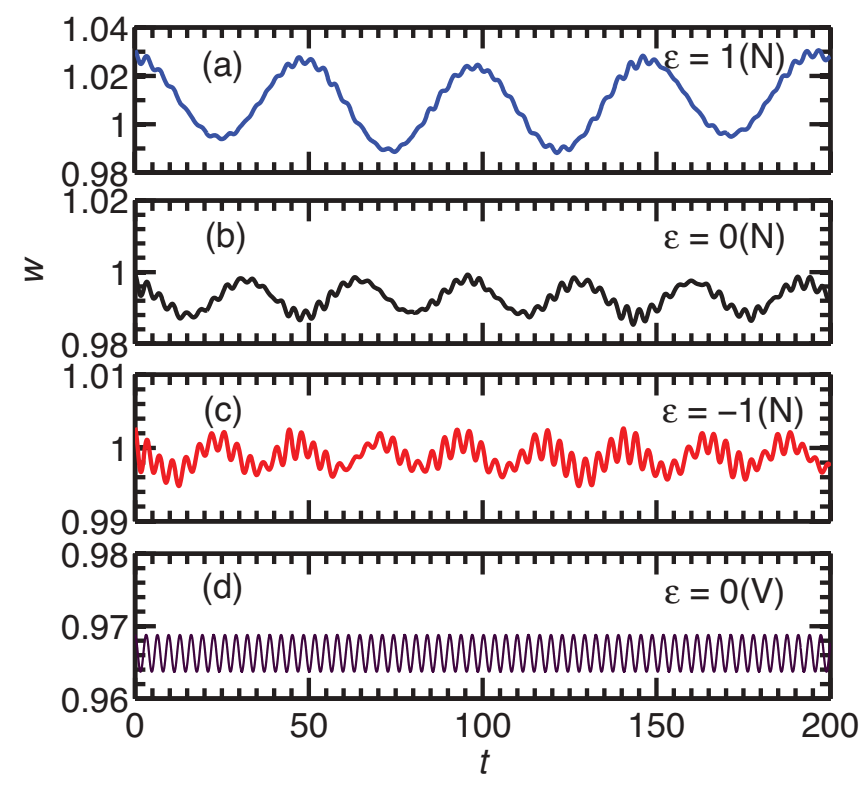

FIG. 6. (Color online) Numerical $(\mathrm{N})$ result for dimensionless pulse width $w$ of the localized state vs dimensionless time $t$ during breathing oscillation initiated by suddenly changing the strength of the secondary lattice $s_{2}$ from 3 to 3.5 for $\varepsilon=$ (a) 1 , (b) 0 , and (c) -1 . We also show the variational result $(\mathrm{V})$ of pulse width $w$ from a solution of Eqs. (11) and (12) with condition $w(t=0)=0.9688$ and $\beta(t=0)=0$. 
Gaussian part. The variational frequency as obtained from Fig. $6(d)$ is found to be identical with the frequency of modulation of the numerical width, which confirms that the modulation in Figs. 6(a), 6(b), and 6(c) is coming from the oscillation of the central part of the condensate. The renewed oscillation in Figs. 5 and 6 confirms the stability of the stationary localized BECs.

\section{SUMMARY}

In this paper, using the numerical and variational solution of the time-dependent GP equation, we studied the stationary and dynamic characteristics of a cigar-shaped localized BEC with spatially inhomogeneous nonlinearity in a bichromatic quasiperiodic 1D OL potential. This investigation reveals that the spatially inhomogeneous nonlinearity produces a pseudopotential which changes the strength of the disorder and the height of the quasiperiodic effective potential felt by the localized BEC. With a larger spatially modulated coefficient $\varepsilon$, the localization length and Hamiltonian will be larger.
We also study the stability of the stationary localized state using the linear stability analysis and find it is dynamically stable under small perturbations. The stability is also verified by numerical simulation. In respect to dynamics, we investigate the location oscillation (oscillation of the center) and breathing oscillation of the localized BEC, and find that both oscillations are quasiperiodic because of the quasiperiodic effective potential. The frequency of quasiperiodic oscillations of the center of the BEC increases as $\varepsilon$ increases. For the breathing oscillations, the two exponential tails also are symmetric around the center at $x=0$. The present study is useful for an understanding of the statics and dynamics of Anderson localization and for planning new experiments with collisionally inhomogeneous BEC.

\section{ACKNOWLEDGMENTS}

FAPESP (Brazil) and CNPq (Brazil) provided partial support.
[1] P. W. Anderson, Phys. Rev. 109, 1492 (1958).

[2] D. S. Wiersma et al., Nature (London) 390, 671 (1997); F. Scheffold et al., ibid. 398, 206 (1999); R. Dalichaouch et al., Nature (London) 354, 53 (1991); A. A. Chabanov et al., Nature (London) 404, 850 (2000).

[3] R. L. Weaver, Wave Motion 12, 129 (1990).

[4] J. Billy et al., Nature (London) 453, 891 (2008).

[5] G. Roati et al., Nature (London) 453, 895 (2008).

[6] L. Fallani, C. Fort, and M. Inguscio, Adv. At. Mol. Opt. Phys. 56, 119 (2008); J. E. Lye, L. Fallani, M. Modugno, D. S. Wiersma, C. Fort, and M. Inguscio, Phys. Rev. Lett. 95, 070401 (2005).

[7] L. Fallani, J. E. Lye, V. Guarrera, C. Fort, and M. Inguscio, Phys. Rev. Lett. 98, 130404 (2007).

[8] P. G. Harper, Proc. Phys. Soc. London, Sect. A 68, 874 (1955).

[9] S. Aubry and G. André, Ann. Israel. Phys. Soc. 3, 133 (1980).

[10] L. Sanchez-Palencia, D. Clément, P. Lugan, P. Bouyer, G. V. Shlyapnikov, and A. Aspect, Phys. Rev. Lett. 98, 210401 (2007); D. Clément, A. F. Varón, M. Hugbart, J. A. Retter, P. Bouyer, L. Sanchez-Palencia, D. M. Gangardt, G. V. Shlyapnikov, and A. Aspect, ibid. 95, 170409 (2005); T. Paul, M. Albert, P. Schlagheck, P. Leboeuf, and N. Pavloff, Phys. Rev. A 80, 033615 (2009); T. Paul, P. Schlagheck, P. Leboeuf, and N. Pavloff, Phys. Rev. Lett. 98, 210602 (2007).

[11] G. Roux, T. Barthel, I. P. McCulloch, C. Kollath, U. Schollwock, and T. Giamarchi, Phys. Rev. A 78, 023628 (2008); B. Damski, J. Zakrzewski, L. Santos, P. Zoller, and M. Lewenstein, Phys. Rev. Lett. 91, 080403 (2003); T. Schulte, S. Drenkelforth, J. Kruse, W. Ertmer, J. Arlt, K. Sacha, J. Zakrzewski, and M. Lewenstein, ibid. 95, 170411 (2005); T. Roscilde, Phys. Rev. A 77, 063605 (2008); X. Deng et al., Eur. Phys. J. B 68, 435 (2009).

[12] J. Chabé, G. Lemarié, B. Grémaud, D. Delande, P. Szriftgiser, and J. C. Garreau, Phys. Rev. Lett. 101, 255702 (2008); E. E. Edwards, M. Beeler, T. Hong, and S. L. Rolston, ibid. 101, 260402 (2008).

[13] R. C. Kuhn, C. Miniatura, D. Delande, O. Sigwarth, and C. A. Muller, Phys. Rev. Lett. 95, 250403 (2005); S. E. Skipetrov,
A. Minguzzi, B. A. van Tiggelen, and B. Shapiro, ibid. 100, 165301 (2008); E. Abrahams, P. W. Anderson, D. C. Licciardello, and T. V. Ramakrishnan, Phys. Rev. Lett. 42, 673 (1979).

[14] A. S. Pikovsky and D. L. Shepelyansky, Phys. Rev. Lett. 100, 094101 (2008); S. Flach, D. O. Krimer, and Ch. Skokos, ibid. 102, 024101 (2009); I. García-Mata and D. L. Shepelyansky, Phys. Rev. E 79, 026205 (2009); Ch. Skokos, D. O. Krimer, S. Komineas, and S. Flach, ibid. 79, 056211 (2009); P. Lugan, D. Clement, P. Bouyer, A. Aspect, and L. Sanchez-Palencia, Phys. Rev. Lett. 99, 180402 (2007); J. E. Lye, L. Fallani, C. Fort, V. Guarrera, M. Modugno, D. S. Wiersma, and M. Inguscio, Phys. Rev. A 75, 061603(R) (2007).

[15] S. K. Adhikari and L. Salasnich, Phys. Rev. A 80, 023606 (2009); Y. Cheng and S. K. Adhikari, ibid. 81, 023620 (2010); 82, 013631 (2010); Laser Phys. Lett. 7, 824 (2010); S. K. Adhikari, Phys. Rev. A 81, 043636 (2010).

[16] S. Inouye et al., Nature (London) 392, 151 (1998).

[17] M. Theis, G. Thalhammer, K. Winkler, M. Hellwig, G. Ruff, R. Grimm, and J. H. Denschlag, Phys. Rev. Lett. 93, 123001 (2004).

[18] G. Roati, M. Zaccanti, C. D’Errico, J. Catani, M. Modugno, A. Simoni, M. Inguscio, and G. Modugno, Phys. Rev. Lett. 99, 010403 (2007); P. Zhang, P. Naidon, and M. Ueda, ibid. 103, 133202 (2009).

[19] P. O. Fedichev, Yu. Kagan, G. V. Shlyapnikov, and J. T. M. Walraven, Phys. Rev. Lett. 77, 2913 (1996).

[20] H. Sakaguchi and B. A. Malomed, Phys. Rev. E 72, 046610 (2005)

[21] J. Belmonte-Beitia, V. M. Pérez-García, V. Vekslerchik, and V. V. Konotop, Phys. Rev. Lett. 100, 164102 (2008); A. T. Avelar, D. Bazeia, and W. B. Cardoso, Phys. Rev. E 79, 025602(R) (2009); J. Belmonte-Beitia and J. Cuevas, J. Phys. A 42, 165201 (2009).

[22] F. Kh. Abdullaev, R. M. Galimzyanov, M. Brtka, and L. Tomio, Phys. Rev. E 79, 056220 (2009); F. Kh. Abdullaev, A. Gammal, M. Salerno, and L. Tomio, Phys. Rev. A 77, 023615 (2008); 
F. Abdullaev, A. Gammal, and L. Tomio, J. Phys. B 37, 635 (2004).

[23] R. Yamazaki, S. Taie, S. Sugawa, and Y. Takahashi, Phys. Rev. Lett. 105, 050405 (2010).

[24] P. Niarchou, G. Theocharis, P. G. Kevrekidis, P. Schmelcher, and D. J. Frantzeskakis, Phys. Rev. A 76, 023615 (2007); G. Theocharis, P. Schmelcher, P. G. Kevrekidis, and D. J. Frantzeskakis, ibid. 72, 033614 (2005); A. S. Rodrigues, P. G. Kevrekidis, M. A. Porter, D. J. Frantzeskakis, P. Schmelcher, and A. R. Bishop, ibid. 78, 013611 (2008); V. M. Pérez-García and R. Pardo, Physica D 238, 1352 (2009).

[25] G. Theocharis, P. Schmelcher, P. G. Kevrekidis, and D. J. Frantzeskakis, Phys. Rev. A 74, 053614 (2006); J. Garnier and F. Kh. Abdullaev, ibid. 74, 013604 (2006); F. Kh. Abdullaev, A. Gammal, H. L. F. da Luz, and L. Tomio, ibid. 76, 043611 (2007).

[26] F. Abdullaev, A. Abdumalikov, and R. Galimzyanov, Phys. Lett. A 367, 149 (2007).
[27] C. A. G. Buitrago and S. K. Adhikari, J. Phys. B 42, 215306 (2009); L. Salasnich, A. Parola, and L. Reatto, Phys. Rev. A 65, 043614 (2002); S. K. Adhikari and B. A. Malomed, ibid. 79, 015602 (2009); Y. Cheng, J. Phys. B 42, 205005 (2009); P. Muruganandam and S. K. Adhikari, Comput. Phys. Commun. 180, 1888 (2009).

[28] P. J. Y. Louis, E. A. Ostrovskaya, C. M. Savage, and Y. S. Kivshar, Phys. Rev. A 67, 013602 (2003).

[29] M. Modugno, New J. Phys. 11, 033023 (2009); M. Larcher, F. Dalfovo, and M. Modugno, Phys. Rev. A 80, 053606 (2009).

[30] R. Scharf and A. R. Bishop, Phys. Rev. E 47, 1375 (1993).

[31] V. M. Pérez-García, H. Michinel, J. I. Cirac, M. Lewenstein, and P. Zoller, Phys. Rev. A 56, 1424 (1997).

[32] Y. Cheng, R. Gong, and H. Li, Opt. Express 14, 3594 (2006).

[33] T. Mayteevarunyoo, B. A. Malomed, and G. Dong, Phys. Rev. A 78, 053601 (2008).

[34] J. Mun, P. Medley, G. K. Campbell, L. G. Marcassa, D. E. Pritchard, and W. Ketterle, Phys. Rev. Lett. 99, 150604 (2007). 